\title{
Maculopapular rash in COVID-19 patient treated with lopinavir/ritonavir
}

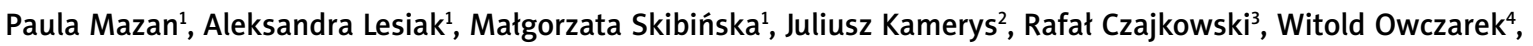 \\ Joanna Narbutt ${ }^{1}$
}

1Department of Dermatology, Paediatric Dermatology and Oncology, Medical University of Lodz, Lodz, Poland 2Department of Infectious Diseases and Hepatology, Medical University of Lodz, Lodz, Poland ${ }^{3}$ Department of Dermatology, Sexually Transmitted Disorders and Immunodermatology, Collegium Medicum in Bydgoszcz, Nicolaus Copernicus University in Torun, Poland

${ }^{4}$ Department of Dermatology, Warsaw Medical Institute, Central Clinical Hospital of the Ministry of Defence, Warsaw, Poland

Adv Dermatol Allergol 2020; XXXVII (3): 435-437 DOI: https://doi.org/10.5114/ada.2020.95029

Exanthematous drug eruption, also called morbiliform drug eruption is a type IV immune reaction, mediated by cytotoxic T-cells. It is characterized by pruritic erythematous macules or papules, evolving rapidly and typically presenting 5 days to 3 weeks after the new drug administration [1]. The most common causes are antibiotics, anti-epileptics, allopurinol, non-steroidal anti-inflammatory drugs (NSAIDs), anxiolytics, antihypertensives, diuretics and antiretroviral drugs. Antiretroviral agents used to treat HIV-positive patients are common drugs with multiple and frequent cutaneous manifestations, which were observed in the past decades [2]. The morbiliform eruption is the most common type of reaction after initiation of antiretroviral treatment [3]. There have been reports of pruriginous exanthematous drug eruptions caused especially by a combination of lopinavir/ritonavir [4]. We present a 35-year-old male patient suspected of undergoing the SARS-CoV-2 infection who was admitted to the Covid-19 unit of our hospital. He reported no symptoms, nor had a history of travels abroad or exposure to patients infected or suspected of contagious COVID-19. In the week preceding the admission the patient was diagnosed with optic neuritis and the treatment prescribed on an outpatient basis included $16 \mathrm{mg}$ of methylprednisolone. On the follow-up, due to patient management associated with the coronavirus pandemic, a rapid test for COVID-19 was performed; the test was positive. The patient was admitted to the COVID-19 ward of our hospital and pharyngeal swab specimens were collected for the SARS-CoV-2 viral nucleic acid detection using real-time reverse transcriptasepolymerase chain reaction (RT-PCR) assay, confirming the diagnosis. He did not have any other abnormalities in laboratory tests (laboratory results were within reference ranges). The patient was hospitalized in the isolation unit and treated with oral lopinavir/ritonavir 400/100 BID. There was an assumption that optic neuritis could have been an unspecific symptom of the coronavirus infection. After two negative RT-PCR tests the patient was considered recovered from COVID-19 disease.

Following 10 days of lopinavir/ritonavir administration, the patient developed an itchy, maculopapular rash while being hospitalized. Initially the lesions appeared on the skin of the trunk, after $24 \mathrm{~h}$ they spread to the upper extremities. Dermatological examination revealed non-tender erythematous macules and papules, bilateral and symmetrical in distribution, localized on the skin of the neck, trunk and arms (Figures 1 A, B). The patient had no history of contact dermatitis, previous adverse drug reactions, or other hypersensitivity reactions. Considering prior treatment with methylprednisolone, it was decided to increase the dose of systemic corticosteroids and initiate topical treatment with corticosteroids and systemic antihistamines. During 10-day follow-up skin lesions disappeared almost completely. The patient is under ophthalmological care, where improvement of vision is being observed.

A maculopapular rash is a skin condition that often occurs in patients with bacterial and viral infections including scarlet fever, measles, Ebola virus disease, rubella, erythrovirus (parvovirus B19), Dengue fever, chikungunya, zika, smallpox, varicella, heat rash and hypersensitivity reactions (exanthematous drug reactions) [5]. While bacterial and viral infections are a well-known cause of maculopapular rash there are some speculations about cutaneous manifestations associated with

Address for correspondence: Paula Mazan MD, Department of Dermatology, Paediatric Dermatology and Oncology,

Medical University of Lodz, 1/5 Kniaziewicza St, 91-347 Lodz, Poland, phone: + 48793552 532, e-mail: paula.mazan.pm@gmail.com Received: 16.04.2020, accepted: 27.04.2020 


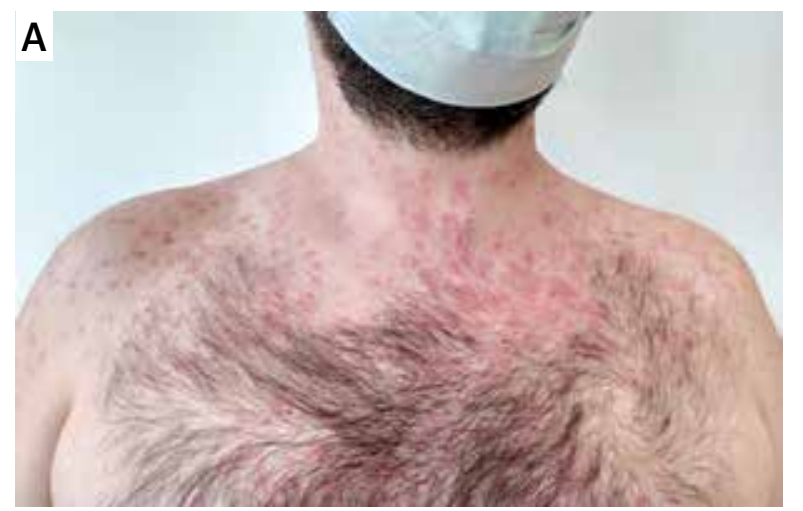

Figure 1. A, B - Erythematous macules and papules located on the skin of the neck, trunk and arms

COVID-19. The data collected by dermatologists analysing skin lesions in 88 Italian COVID-19 patients show that one-fifth of them developed cutaneous manifestations. In 8 patients they were present on admission to the hospital and in 10 patients they were observed during follow-up and in most cases they were reported as erythematous rash. Other cutaneous manifestations included urticaria (3 patients) and chickenpox-like vesicles (1 patient). Skin lesions were mainly localized on the trunk and healed within a few days. No correlation between disease's severity and skin manifestations was observed [6]. In some cases, symptoms of COVID-19 may mimic rashes in other viral infections resulting in misdiagnosis. A case of the patient with skin rash, petechiae and a low platelet count initially diagnosed as a Denga fever was described. When he developed respiratory problems and after ruling out other common viral infections, the final diagnosis of the COVID-19 infection was confirmed by RT-PCR [7].

Vasculitis, transient livedoid eruptions and cyanosis have also been reported as potential skin manifestations of COVID-19. However, more data need to be obtained to define cutaneous involvement in the course of the SARS-CoV-2 infection. The possibility of skin lesions as the only COVID-19 symptom should be considered by practitioners to avoid transmission of the coronavirus. Worldwide there is a few accepted COVID-19 treatment regimens including antiviral and immune modulating therapies as well as novel agents available in clinical trials [8]. Few case reports of maculopapular rash associated with lopinavir/ritonavir administration have been published. Skin lesions occurred approximately 10 days after treatment with lopinavir/ritonavir, improved on withdrawal and relapsed following its reintro-

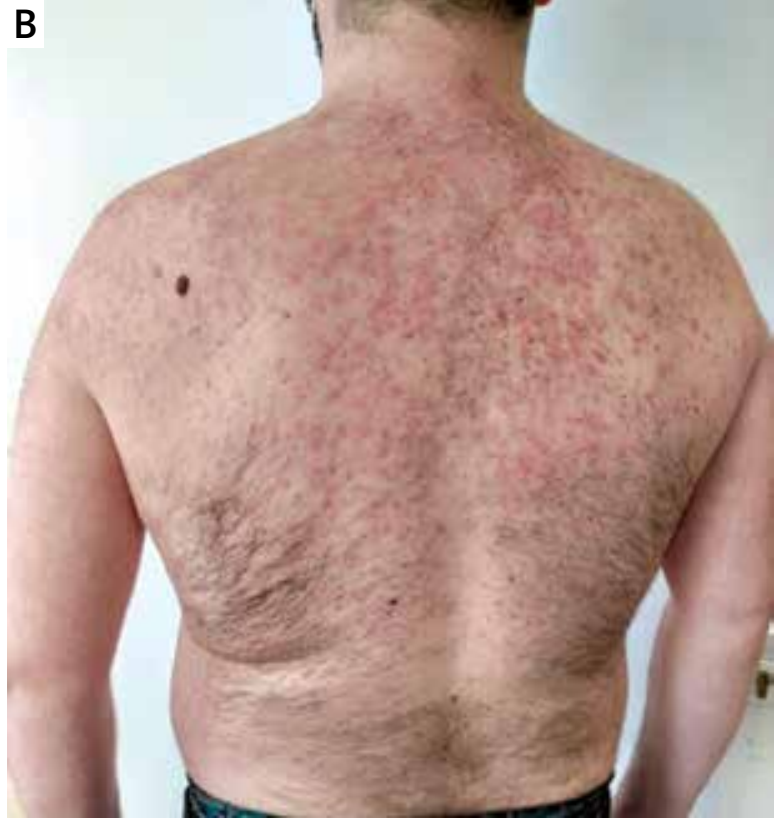

duction [9]. Other protease inhibitors such as darunavir, nucleoside reverse transcriptase inhibitor - abacavir or non-nucleoside analogue reverse transcriptase inhibitor called efavirenz have also been reported as a cause of maculopapular rash [3, 4]. Based on current reports we cannot state with certainty that the maculopapular rash observed in our patient is only drug-induced, especially due to the unspecific course of the infection in this patient. There is a suspicion that COVID19 could be a cofactor for maculopapular rash. However, the role of SARS-CoV-2 in inducing skin lesions is still uncertain and needs to be clarified based on further research and observations.

\section{Acknowledgments}

This research was funded by statutory activities of the Medical University of Lodz (no. 503/5-064-04/503-01).

\section{Conflict of interest}

The authors declare no conflict of interest.

\section{References}

1. Stern R. Exanthematous drug eruptions. N Engl J Med 2012; 366: 2492-501.

2. Introcaso CE, Hines JM, Kovarik CL. Cutaneous toxicities of antiretroviral therapy for HIV: part I. Lipodystrophy syndrome, nucleoside reverse transcriptase inhibitors, and protease inhibitors. J Am Acad Dermatol 2010; 63: 549-61.

3. Borrás-Blasco J, Navarro-Ruiz A, Borrás C, Casterá E. Adverse cutaneous reactions associated with the newest antiretroviral drugs in patients with human immunodeficiency virus infection. J Antimicrob Chemother 2008; 62: 879-88. 
4. Blanes M, Belinchón I, Portilla J. Cutaneous drug reactions in HIV-infected patients in the HAART era. Actas Dermosifiliog 2009; 100: 253-65.

5. Muzumdar S, Rothe MJ, Grant-Kels JM. The rash with maculopapules and fever in adults. Lin Dermatol 2019; 37: 109-18.

6. Recalcati S. Cutaneous manifestations in COVID-19: a first perspective. J Eur Acad Dermatol Venereol 2020; 34: e212-3.

7. Joob B, Wiwanitkit V. COVID-19 can present with a rash and be mistaken for Dengue. J Am Acad Dermatol 2020; 82: e177.

8. Barlow A, Landolf KM, Barlow B, et al. Review of emerging pharmacotherapy for the treatment of coronavirus disease 2019. Pharmacotherapy 2020 Apr 7. doi: 10.1002/phar.2398.

9. Calista D. Maculo-papular rash induced by lopinavir/ritonavir. Eur J Dermatol 2005; 15: 97-8. 